\title{
DETERMINACIÓN DE LOS PRECIOS DE LOS APARTAMENTOS TURÍSTICOS AIRBNB EN MÁLAGA. UNA APROXIMACIÓN ESPACIAL
}

\author{
JUAN GABRIEL GONZÁLEZ MORALES \\ Departamento Economía Financiera y Contabilidad, UNIVERSIDAD DE GRANADA, ESPAÑA \\ e-mail: juangonzalezm@ugr.es \\ JORGE CHICA OLMO \\ Departamento Métodos Cuantitativos para la Economía y la Empresa, UNIVERSIDAD DE GRANADA, \\ ESPAÑA \\ e-mail: jchica@ugr.es \\ JOSÉ LUIS ZAFRA GÓMEZ \\ Departamento Economía Financiera y Contabilidad, UNIVERSIDAD DE GRANADA, ESPAÑA \\ e-mail:jlzafra@ugr.es
}

\section{RESUMEN}

En este trabajo se presentan los principales resultados de la modelización hedónica del precio de los apartamentos turísticos localizados en la ciudad de Málaga dentro del marco incipiente conocido como sharing economy. Para ello se dispone de información de la empresa de apartamentos turísticos Airbnb, que es una plataforma de alquiler de alojamientos que está presente en todo el mundo. En este trabajo se estimará un modelo de precios hedónicos que tiene en consideración características estructurales (número de camas, dormitorios, numero de baños...) y algunas características específicas que se conocen como amenities (piscina, wifi, ascensor etc.). Todo esto nos permitirá determinar algunos de los principales factores que determinan el precio de alquiler del alojamiento en dicha ciudad. De esta forma ofreceremos finalmente una vía de análisis espacial de dicho fenómeno que no hemos visto considerada en otros trabajos de este tipo.

Palabras clave: Sharing economy, Hedonic price, Modelo hedónico de precios, Airbnb

\section{Determination of the prices of AIRBNB tourist apartments in Malaga. A space approach}

\begin{abstract}
In this paper we present the main results of tourism apartment hedonic price modelization located in Malaga, under the incipient sharing economy framework. For this, information is available about the Company of touristic aparments Airbnb, which is a rental platform for accommodation that is located around the world. In this paper, a hedonic price model is estimated that takes into account structural characteristics (number of beds, bedrooms, number of bathrooms...) and some specific features that are known as amenities (pool, wifi, elevator etc.). All of this will allow us to determine some $f$ the main factors that determine the rental price of the accommodation in said city. In this way, we finally show a way of spatial analysis of this phenomenon that we have not seen before in other works of this type.
\end{abstract}

Keywords: Sharing economy, Hedonic price, Hedonic price model, Airbnb

Clasificación JEL: C51 


\section{INTRODUCCIÓN}

A nivel mundial, el turismo ha crecido a gran escala. Las mejoras en accesibilidad en los transportes han supuesto que desde el año 2000 la cifra de turistas fuera de 680 millones y en 2017 de 1322 millones (World Tourism Organization (UNWTO), 2018). Este aumento a nivel internacional no ha sido indiferente dentro de las fronteras españolas. En nuestro país, se ha seguido la misma tendencia, aumentando en más de 4 millones el número de turistas en el mismo periodo (Gobierno de España. Ministerio de Energía, 2018).

Esto ha dado lugar a la inversión en infraestructuras hoteleras, aparición de nuevos destinos turísticos y nuevas rutas de transporte hacia los mismos. Sin embargo, dada la gran demanda en términos de alojamiento se ha desarrollado una tendencia (bastante asentada) en torno a los alojamientos compartidos. La aparición de los hostels (hostales) y B\&B (bed and breakfast) junto con el abaratamiento de los costes en el viaje, ha supuesto que un mayor público con menor poder adquisitivo (especialmente los jóvenes) tengan acceso a la posibilidad de viajar.

Con el tiempo, este concepto ha ido evolucionando y ya no solo son profesionales que gestionan edificios completos de este tipo de alojamiento los que compiten en el mercado. En 2008 nace la idea de crear una plataforma donde tanto particulares como profesionales puedan ofrecer sus alojamientos (viviendas) o habitaciones en sus viviendas, esta se llama Airbnb. Bajo la filosofía de sharing economy, la empresa cuenta ya con más de 10.000 .000 de hospedajes realizados y está presente en más de 30.000 ciudades de todo el globo (Inside Airbnb, 2017), está valorada en más de 10 millones de dólares y está considerada como la empresa de alojamiento más grande del mundo. Su evolución en los últimos años ha sido de carácter exponencial superando a compañías como Intercontinental o Hyatt en cuanto a valor se refiere (Libre Mercado, 2015). Sin embargo, en los últimos años el debate ha estado en torno a la influencia de esta plataforma sobre la industria hotelera debido a su fuerte competencia en precios, así como la localización de los alojamientos en lugares dónde (al tratarse de viviendas particulares) por motivos estructurales, el sector hotelero no tiene acceso. Los alojamientos compartidos se presentan como un importante competidor de los métodos tradicionales hoteleros. Uno de los aspectos de interés de este tipo competencia son los precios de sus productos. Por lo tanto, se hace conveniente determinar los principales factores que rigen dichos precios. Para ello, los modelos hedónicos son los métodos adecuados para determinar los factores que determinan los precios de los alojamientos compartidos.

La estructura de este trabajo es la siguiente: En primer lugar, se realiza una revisión bibliográfica en la que se consideran con especial interés los conceptos de sharing economy, el modelo hedónico y los principales factores que lo determinan. A continuación, se presentan los principales resultados del estudio del precio de los apartamentos turísticos en Málaga. Se finaliza con las conclusiones y las líneas futuras de investigación.

\section{REVISIÓN BIBLIOGRÁFICA}

\subsection{Sharing Economy}

Sharing Economy (economía colaborativa) es un concepto que está presente actualmente en la actividad económica y que cada vez va ganando más fuerza. Este concepto se basa en la red como principal fuente de difusión, dónde existen plataformas en las que los usuarios ofrecen sus propios recursos inactivos en alquiler para aquellas personas que lo soliciten. Autores como Yoonjoung Heo (2016) realizan una definición más estricta centrando este concepto como: "un sistema económico donde recursos y servicios son compartidos entre individuos privados, algunos gratuitos y otros con un precio asequible, bajo el respaldo de internet", pero ambos coinciden en la importancia de la red para el crecimiento de este movimiento. De hecho, Belk (2014) en su estudio teórico refleja dos conceptos en los que se basa la economía colaborativa; el uso temporal en no propiedad a bienes y servicios de consumo y lo que se conoce como La Web 2.0. En definitiva, este concepto se ha convertido en un modelo de negocio competitivo que supone un 
verdadero reto para los servicios tradicionales por lo que es plausible su análisis detallado (Möhlmann, 2015).

En la literatura, el concepto de economía colaborativa ha sido motivo de estudio desde muchas vertientes. Bing, Qiang, y Rob (2016) realizan una definición seminal del concepto, estableciendo una relación directa con la investigación en el ámbito turístico. Para el autor, toda la literatura acerca del mismo está dividida en torno a tres aspectos: una aproximación psicológica, ámbito legal y financiero y lo relacionado con las actividades presentes en sharing economy.

Siguiendo esta clasificación, algunos trabajos como Hamari, Sjöklint, \& Ukkonen (2016) realizan un análisis desde la perspectiva psicológica del motivo de elección por parte de los usuarios hacia la economía colaborativa. En él, se plantean aspectos como motivación intrínseca (placer), extrínseca (reputación) y actitud positiva como elementos que influyen en la actitud de entrar en el mercado de la misma. Otros como Möhlmann (2015) van más allá y plantean distintos factores para determinar la probabilidad de que los usuarios de plataformas colaborativas repitan su experiencia, especificando qué factores como la familiaridad, satisfacción y utilidad de la estancia son estadísticamente robustos. De hecho, Makarand, Suess, \& Xinran (2017) aportan el concepto de "experiencescape" que hace referencia a las perspectivas personales e intangibles que hacen que en el contexto de sharing economy los anfitriones faciliten experiencias extraordinarias a los huéspedes.

En el aspecto legal y financiero, diversos estudios han profundizado en la normativa vigente de las relaciones entre usuarios (normativa existente no muy clara en el concepto regalo o contrato) e incluso regulaciones por parte del gobierno (Thorne \& Quinn, 2017; Morgan \& Kuch, 2015), mientras que otros han hecho mayor hincapié en analizar a los sectores diferenciados de sharing economy y mediante la perspectiva legal, aportar directrices en las que actuar a las empresas tradicionales (Cusumano, 2015). De hecho, Miller (2016) desarrolla una revisión de la legislación en economía colaborativa para luego analizar el caso de plataformas de alojamiento (Airbnb) en San Francisco, California y Oregón, proporcionando una respuesta diferente a las estrategias tradicionales basada en los mercados y los derechos de distribución transferibles (más adecuado para internalizar las externalidades de los alquileres a corto plazo).

Por último, el análisis de las actividades. En este aspecto se han realizado muchos trabajos empíricos utilizando como referencia las diferentes plataformas de sharing economy tanto en su vertiente de transporte (Uber y Blablacar) como en la de alojamiento (Airbnb). Para el caso de Uber, se ha estudiado las características de este tipo de plataforma así como las implicaciones y consecuencias que tienen sobre su homólogo público (Leighton, 2016), además de encasillarlo en el concepto de innovación disruptiva (Schwalbe, 2018), donde se considera el mercado como un proceso dinámico y se centra principalmente en los individuos. Para Blablacar en cambio, el análisis se ha llevado a cabo en torno a las características de los usuarios y de los conductores según sus características socio demográficas (Shaheen, Stocker, \& Mundler, 2017). Referidos a la categoría de alojamiento, también Airbnb ha sido catalogado como innovación disruptiva, delimitando los canales de distribución y los principales afectados, así como el modelo de negocio de la compañía. (Guttentag, 2015). Por otro lado, se ha analizado también tanto a los agentes implicados como al proceso de transacción. Desde el lado de los anfitriones se ha puntualizado una diferencia notable en los resultados entre los anunciantes que son profesionales con los que no (Jun, Moreno, \& Zhang, 2015), así como los factores que determinan que a un anfitrión se le encasille en la categoría de profesional (Gunter, 2018). Desde el punto de vista del proceso, se ha demostrado que el método de transacción basado en la confianza implica efectos indirectos en la posibilidad de repetir en el uso de la plataforma, (Liang, Choi, \& Joppe, 2018), mientras que So, Oh, \& Min (2018) enfatizan en determinar que los precios más bajos, beneficios económicos o el valor percibido son los factores principales que hacen que el huésped se decante por la opción Airbnb.

Además de todos estos estudios orientados a los agentes y el proceso de Airbnb, otros autores se han focalizado en la influencia de esta plataforma sobre el sector turístico, ya que Airbnb ha supuesto toda una revolución en el mismo. La industria hotelera ha visto como una plataforma diferente, basada en sharing economy ha ganado una fuerte cuota de mercado con políticas de precios bastante diferentes a las suyas. No solo propone precios más asequibles, sino que hay una 
mayor facilidad para que las características del alojamiento se ajusten a un público mayor (Gutiérrez, García-Palomares, Romanillos, \& Salas-Olmedo, 2017). Mientras que el sector hotelero presenta una mayor homogeneidad en su oferta, determinado en gran medida por un sistema de calificación basado en estrellas, en esta plataforma hay una mayor heterogeneidad (Guttentag, 2015). Se encuentran diversidad de ofertas de alojamiento, desde habitaciones en viviendas habituales, hasta villas con capacidad para 20 personas y donde las valoraciones por parte de los usuarios anteriores juegan un papel importante en el criterio de decisión de los nuevos inquilinos. De hecho, Yoonjoung Heo (2016) en su estudio defiende que los turistas que utilizan las plataformas de economía colaborativa se enfocan más en la valoración de las experiencias comparado con el turismo tradicional.

Otros, como es el caso de Zervas, Proserpio, \& Byers (2017), han determinado modelos para cuantificar que (en Texas) un incremento del 1\% de alojamientos tipo Airbnb suponen un $0.05 \%$ de decrecimiento de los beneficios trimestrales de los hoteles de la zona, o la investigación realizada por Guttentag \& Smith (2017) que mediante un estudio de información primaria señalan que el $64,8 \%$ de los encuestados usan Airbnb como sustituto directo de los hoteles, más concretamente de un rango medio ( 3 estrellas). Por otro lado, esta relación ha sido estudiada desde otro punto de vista por autores como Gutiérrez, García-Palomares, Romanillos, \& Salas-Olmedo (2017). En su trabajo estudian los patrones espaciales de la distribución hotelera y los Airbnb con el objetivo de analizar la distribución de los mismos y aplicar modelos de regresión para la densidad hotelera y de Airbnb.

\subsection{Precio Hedónico}

Los modelos hedónicos de precios se caracterizan como una solución a los problemas de equilibrio de la economía, donde la totalidad de los precios guían tanto al consumidor como al productor en la toma de decisiones dadas esas características (Rosen, 1974). Por tanto se especifica un aspecto muy importante en el establecimiento de los modelos hedónicos de precios, las características que determinan los mismos. En definitiva, el objetivo de un modelo hedónico es determinar el precio implícito de cada uno de los factores que determina el precio del bien.

En nuestro caso, el punto de partida son los modelos de precio hedónicos aplicados a la vivienda, por similitud con el objeto de estudio. Sin embargo, las características son bastante diferentes con los estudios tradicionales de vivienda. Investigaciones como las realizadas por Can (1992); Dubin (1998); Case, Clapp, Dubin, \& Rodríguez (2004) o Chica Olmo, Cano Guervos, \& Chica Olmo (2007); determinan que las características estructurales de la vivienda como el número de habitaciones, el número de baños y la antigüedad son de vital importancia. Para el caso de nuestro estudio, tendremos en cuenta algunas de las características estructurales que ofrecen estos trabajos y aportaremos nuevas categorías de variables

En la literatura, también se han utilizado los modelos hedónicos de precios hoteleros para determinar las características que influyen sobre los precios del sector, agrupándolas en torno a localización, número de estrellas, calidad del servicio y comodidades (Thrane, 2007). Algunos autores como Chen \& Rothschild (2010) analizaron una muestra de 73 hoteles en Taipei y observaron que un conjunto de características como tener TV, internet y gimnasio fueron significativas. Otros como Masiero, Nicolau, \& Law (2015) añadieron conceptos nuevos como la inclusión del tipo de hotel, los canales de venta del mismo e incluso los canales de difusión utilizados, además de la temporada de viaje. A partir de ahí, se han desarrollado investigaciones orientadas a Airbnb, basadas precisamente en la modelización hedónica de precios hoteleros. En este contexto Wang \& Nicolau (2016) estudiaron mediante una regresión por cuartiles una muestra de 33 ciudades diferentes, aplicando características determinantes del precio en torno a factores de calidad del alojamiento, comodidades y servicios, características de la propiedad, factores externos y algunos factores relacionados con la localización.

En la Tabla I se muestra un resumen de los principales trabajos considerados en la revisión bibliográfica. Teniendo en cuenta ésta, nuestro estudio tiene cabida en la literatura sobre el tema. Diversos autores han estudiado el concepto del modelo hedónico de precios para sharing 
economy (Airbnb). Sin embargo, en este estudio vamos a aportar resultados para un área en concreto (Málaga).

TABLA I

Principales estudios en Sharing Economy y Precio Hedónico

\begin{tabular}{|c|c|c|c|c|}
\hline CAMPO & AUTOR & REGIÓN (n) & MÉTODO & CONCLUSIONES \\
\hline $\begin{array}{l}\text { Sharing } \\
\text { economy }\end{array}$ & $\begin{array}{l}\text { Möhlmann, } \\
2015\end{array}$ & $\begin{array}{l}236 \text { encuestas a } \\
\text { usuarios de } \\
\text { "Car2go" y } 187 \text { a } \\
\text { usuarios de Airbnb. }\end{array}$ & $\begin{array}{l}\text { Mínimos cuadrados } \\
\text { parciales para ambas } \\
\text { muestras por } \\
\text { separado. }\end{array}$ & $\begin{array}{l}\text { Similitud para ambos estudios en las } \\
\text { variables que influyen en la decisión } \\
\text { de elegir sharing economy. (ahorro } \\
\text { costes, calidad del servicio, confianza } \\
\text { y utilidad). }\end{array}$ \\
\hline $\begin{array}{l}\text { Sharing } \\
\text { economy }\end{array}$ & $\begin{array}{l}\text { Guttentag \& } \\
\text { Smith, } 2017\end{array}$ & $\begin{array}{l}844 \text { encuestas on- } \\
\text { line centrado en su } \\
\text { mayoría en regiones } \\
\text { canadienses. }\end{array}$ & $\begin{array}{l}\text { Mínimos cuadrados } \\
\text { ordinarios (MCO) }\end{array}$ & $\begin{array}{l}\text { Los huéspedes utilizan Airbnb como } \\
\text { sustituto a los hoteles } \\
\text { convencionales, especialmente los } \\
\text { de medio rango, se considera el } \\
\text { concepto de innovación disruptiva. }\end{array}$ \\
\hline $\begin{array}{l}\text { Sharing } \\
\text { economy }\end{array}$ & Gunter, & $\begin{array}{l}40.060 \text { registros de } \\
\text { alojamientos de } \\
\text { Airbnb en la Bahía } \\
\text { de San Francisco } \\
\text { (EEUU) }\end{array}$ & $\begin{array}{l}\text { Modelo lineal de } \\
\text { probabilidad binaria } \\
\text { (Probit) }\end{array}$ & $\begin{array}{l}\text { El elemento más importante en la } \\
\text { determinación de Superhost es las } \\
\text { buenas valoraciones, seguido de una } \\
\text { política de cancelación flexible y del } \\
\text { interés del anfitrión. }\end{array}$ \\
\hline $\begin{array}{c}\text { Hedonic } \\
\text { Price }\end{array}$ & Dubin, & $\begin{array}{c}1493 \text { registros de } \\
\text { viviendas de } \\
\text { Baltimore, Maryland } \\
\text { (EEUU) }\end{array}$ & $\begin{array}{l}\text { MCO, MCO con } \\
\text { tendencia y } \\
\text { estimación máximo } \\
\text { verosímil (ML) }\end{array}$ & $\begin{array}{l}\text { Diferentes alternativas para la } \\
\text { inclusión de variables espaciales en la } \\
\text { estimación y predicción de precios de } \\
\text { vivienda. }\end{array}$ \\
\hline $\begin{array}{l}\text { Hedonic } \\
\text { Price- } \\
\text { Sharing } \\
\text { economy }\end{array}$ & $\begin{array}{c}\text { Wang \& } \\
\text { Nicolau, } 2016\end{array}$ & $\begin{array}{l}180.533 \text { registros de } \\
\text { ciudades repartidas } \\
\text { por América, Europa } \\
\text { y Oceanía. }\end{array}$ & $\begin{array}{c}\text { MCO y Regresión } \\
\text { Lineal por Cuartiles } \\
\text { (QR) }\end{array}$ & $\begin{array}{l}\text { Factores que determinan los precios } \\
\text { en sharing economy (Airbnb) } \\
\text { difieren a los hoteleros. }\end{array}$ \\
\hline $\begin{array}{l}\text { Hedonic } \\
\text { Price- } \\
\text { Sharing } \\
\text { economy }\end{array}$ & $\begin{array}{l}\text { Gibbs, Guttenta } \\
\text { g, Gretzel, } \\
\text { Morton, \& } \\
\text { Goodwill, } 2018\end{array}$ & $\begin{array}{l}\text { 15.716 registros de } \\
\text { Airbnb para } 5 \\
\text { ciudades } \\
\text { canadienses }\end{array}$ & $\mathrm{MCO}$ & $\begin{array}{l}\text { Aportación importante a la literatura } \\
\text { acerca de factores específicos de } \\
\text { determinación de precios en sharing } \\
\text { economy. }\end{array}$ \\
\hline
\end{tabular}




\section{DETERMINACIÓN DE PRECIOS EN SHARING ECONOMY EN MÁLAGA}

\subsection{Datos}

La base de datos utilizada ha sido proporcionada por Inside Airbnb, donde se ha elegido la ciudad de Málaga porque es una de las principales ciudades turísticas de la Costa del Sol. Además, se ha tenido en cuenta el conocimiento acerca de la distribución espacial en ese territorio y por el crecimiento exponencial que Airbnb ha experimentado en ella.

Airbnb posee diferentes tipos de alojamiento en su servicio como son el alquiler de habitaciones compartidas, habitaciones privadas y los apartamentos enteros. Dentro de este último, podemos encontrar numerosas categorías como villas, bungalows, chalets etc. Existen estudios como el de Wang \& Nicolau, 2016 y Gibbs, Guttentag, Gretzel, Morton, \& Goodwill (2018) que realizan una inclusión de todos los tipos de alojamientos en el estudio diferenciando entre habitación compartida, habitación privada y apartamento entero. No obstante, las características estructurales por parte de cada una de las categorías son muy diferentes, considerando que las que pueden ofrecer apartamentos enteros no se pueden asemejar a las habitaciones compartidas o incluso a villas. Además, en nuestra muestra las habitaciones compartidas representan solo el $2 \%$ del total, mientras que las habitaciones privadas cerca del $20 \%$.

Por ello, dado el carácter diverso de los datos y con el objetivo de homogeneizar la muestra, hemos trabajado solo con los apartamentos enteros y además que tengan categoría de apartamento en sí (excluyendo las villas, bungalows etc). Esto nos garantiza menor dispersión en los datos y llegar a conclusiones de un tipo de producto concreto. En definitiva, nuestra muestra final es de 2968 registros correspondientes a noviembre de 2017.

\subsection{Variables}

En cuanto a las variables explicativas se refiere, esta base de datos contiene gran cantidad de posibilidades que pueden ser relevantes en el campo de estudio. Siguiendo investigaciones anteriores en este campo como Gutiérrez, García-Palomares, Romanillos, \& Salas-Olmedo (2017) o Wang \& Nicolau (2016) es conveniente diferenciarlas en torno a categorías, con el objetivo de centralizar y comprender mejor los datos. Por ello, se ha realizado una clasificación que se basa en 5 apartados: características estructurales, características del anfitrión, comodidades y servicios, reglas del alojamiento y opiniones/valoraciones.

\subsubsection{Características estructurales}

Otros aspectos a considerar para determinar el precio de alquiler, son las características físicas y la capacidad de hospedaje. A pesar de no contar con variables que en la determinación de precios de la vivienda son clave como la antigüedad (Chica Olmo, Cano Guervos, \& Chica Olmo, 2007), hemos empleado el número de baños (N_baños), dormitorios (N_dormitorios) y camas (N_camas). No hemos incluido el número de personas por problemas de multicolinealidad ya que sería redundante al considerarse que dicha información queda recogida en el número de dormitorios y camas.

\subsubsection{Características del anfitrión}

En esta categoría se han incluido las variables Num_alojam, Busqueda_inst y Superhost. La primera de ellas hace referencia al número de alojamientos que un único anfitrión tiene en la web. Airbnb no tiene límites en este sentido, e incluso para que los anunciantes sean profesionales o particulares. En segundo lugar, la variable Busqueda_inst expresa si el huésped necesita una autorización previa para reservar el alojamiento o puede hacerlo directamente. Esto se debe a la naturaleza de la plataforma (sharing economy) que en su gran mayoría no deja de ser un particular quien ofrece su vivienda y por ello algunos prefieren dar una autorización en función del perfil (reseñas y comentarios) del huésped. 
La última variable denota una categoría de "élite" a los anfitriones, es decir, aquellos que han registrado como mínimo 10 servicios, con un $90 \%$ de valoraciones (o mayor), que hayan recibido 5 estrellas el 80\% de los casos y una ratio de cancelación bajo (Gunter, 2018). Variables como el tiempo de antigüedad del anfitrión, la existencia de foto de perfil del mismo o la identidad verificada no fueron consideradas para el estudio ya que indirectamente la información estaba recogida en estas otras variables.

\subsubsection{Comodidades y servicios}

En esta categoría se reúnen todas aquellas comodidades y servicios que se ofrecen en estos alojamientos. Este término es conocido como "amenities" y es el principal criterio de diferencia (junto con las características estructurales) que distingue los hospedajes. Para la extracción de las mismas se ha analizado una columna donde entrada por entrada, se hacía una descripción de las comodidades que se ofrecían. A partir de ahí, se han creado variables ficticias donde el valor 1 denota que ese alojamiento posee esa "comodidad" en concreto y el valor 0 en caso contrario. Existen una gran cantidad de ellas ya que cada anfitrión puede incluir en su descripción todas las que considere oportuno, sin embargo, se descartaron aquellas que eran muy frecuentes (por ejemplo, TV, aire acondicionado, timbre, etc.) y las que rara vez aparecían (por ejemplo, chimenea, detector de humo etc.). Finalmente, las variables elegidas son: la existencia o no de ascensor (Ascensor), que el alojamiento cuente con piscina (Piscina), conexión inalámbrica (Wifi), parking gratuito (Parking_gratis) y bañera de hidromasaje (Bañera_hidro).

La elección de estas variables no ha sido arbitraria. Tanto en los modelos hedónicos de precios de vivienda como los hoteleros, la presencia de piscina o ascensor suelen actuar como criterio diferenciador mientras que la conexión inalámbrica suele incluirse también, aunque cada vez sea más común que se ofrezca (Wang \& Nicolau, 2016). Por otro lado, el parking gratuito también ha sido incluido en investigaciones recientes (Gibbs, Guttentag, Gretzel, Morton, \& Goodwill, 2018) pero además puede ser un aspecto importante dada las dificultades de aparcamiento que tiene la ciudad de Málaga. Se ha considerado la variable Bañera_hidro porque suponemos que la presencia de bañeras de hidromasaje puede implicar un cierto lujo en el alojamiento y, por tanto, el consiguiente plus en el precio.

\subsubsection{Reglas del alojamiento}

Los anfitriones tienen diferentes reglas en sus alojamientos y eso también queda recogido en el estudio. Al igual que pasaba en la categoría anterior, incluiremos a las mismas como variables ficticias dónde se le asignará valor 1 si está permitido y 0 en el caso contrario. La primera de esas variables es la permisión de fumar (Fumar_sí), que dada la creciente implicación por parte de plataformas de alojamiento a la política "libre de humos" (Kennedy, Douglas, Stehouwer, \& Dawson, 2018) puede existir una penalización en el precio. Además, se ha considerado una segunda variable que recoge el consentimiento de la entrada de mascotas (Mascotas_sí) la cual a priori se cree que puede tener influencia sobre el precio de alquiler.

\subsubsection{Opiniones / valoraciones}

El apartado de opiniones/valoraciones es el único que se centra en el huésped y por consiguiente, de ello depende la reputación del anfitrión en la plataforma. Para reflejarlo se han escogido las variables Valoración_host y Num_reseñas que hacen referencia a los elementos presentes en esta categoría. Los usuarios tienen la oportunidad de valorar tanto globalmente como por servicios el alojamiento en el que se hospedan y eso queda recogido en la variable Valoración_host, en un rango 0-1 dónde 1 significa que están totalmente satisfechos y 0 el caso contrario. También es muy importante tener en cuenta el número de comentarios que se realizan, para ello se ha utilizado la variable Num_reseñas, que representa el número de reseñas que ha tenido el alojamiento. En una plataforma donde la confianza y la reputación es un aspecto clave, es plausible incluir la cantidad de comentarios recibidos por parte de un anfitrión en un modelo hedónico de precios (Abdelaziz, Reed, Tyler, \& Yuqing, 2017). 


\subsection{Análisis Descriptivo}

En la tabla II se muestra un análisis descriptivo de todas las variables que se van a tener en cuenta en este estudio, dividido en categorías como se realizó anteriormente y aportando una explicación breve de las mismas. Comenzando con la variable endógena, si comparamos a Málaga con otras regiones tradicionalmente turistas de sol y playa vemos que el precio medio por noche $(75,29 €)$ es inferior al de ciudades como Barcelona (96€) o Mallorca (143€) siendo en este sentido un destino atractivo en cuanto a coste de alojamiento se refiere.

Con respecto a las variables explicativas comenzaremos con las características estructurales. En la categoría, hay que destacar, que con respecto al número de dormitorios, la media en Barcelona y Mallorca se sitúa en 2,1 y 2,4 mientras que en Málaga es de 1,6. Esto nos aporta información a priori de que en la ciudad de estudio la capacidad de huéspedes por apartamento es menor que la de sus homólogos turísticos. Además, la desviación típica (D.T) nos indica una dispersión en torno a 1 a pesar de encontrarnos con valores máximos de 7, suponiendo bastante homogeneidad en torno a esta característica.

En cuanto a las características del anfitrión, tan solo hay una variable cuantitativa (Num_alojam), el resto son variables binarias, por lo que la información que nos aporta la tabla es en torno a la proporción que tenemos de esa determinada característica en la muestra. Sin embargo, de la variable superhost podemos extraer el grado de competencia y profesionalidad que poseen los anfitriones. Para Málaga es de un 15,44\% mientras que para Barcelona y Mallorca es ligeramente superior (16\% y $19 \%$ respectivamente).

En las dos siguientes categorías (comodidades/servicios y reglas del alojamiento), las variables incluidas son todas dummys por lo que al igual que pasaba anteriormente, la información es en torno a la proporción. En la primera de ellas vemos proporciones muy bajas en torno a Bañera_hidro y Piscina, que si se asocian a precios altos puede ser un factor explicativo del mismo bastante importante. Lo antagónico sucede con Wifi, que con una proporción de casi el $90 \%$ parece que está presente en la mayoría de los alojamientos. En la segunda categoría la interpretación es similar, con proporciones por debajo del $25 \%$. Se espera que la influencia sobre el precio sea significativa.

Con respecto a las opiniones y valoraciones, podemos observar que el grado de retroalimentación por parte de los huéspedes a los anfitriones es bastante alto. El nivel de valoración cercano a 9 puntos sobre 10 y con una media de 20 comentarios por huésped. Sin embargo, existe cierta dispersión sobre todo en el número de comentarios, donde la desviación típica nos señala valores relativamente altos. Esto pude estar ocasionado por la presencia de ciertos anfitriones que poseen gran cantidad de comentarios en sus perfiles y actúen de outliers en nuestra muestra.

\section{RESULTADOS}

Una vez detallada la descripción de los datos y las variables, los siguientes pasos son la especificación, estimación y explotación del modelo hedónico de precios. La especificación funcional del modelo final no ha sido arbitraria. La bibliografía consultada nos indica que una especificación semi-logarítimica es adecuada para analizar este tipo de fenómeno (Chen \& Rothschild, 2010).

$$
\begin{aligned}
& L O G(P R E C I O)=\beta \_0+\beta \_1 * N \_b a n ̃ o s+\beta \_2 * N \_d o r m i t o r i o s+\beta \_3 * N \_c a m a s+\beta \_4 \\
& * N u m_{-} \text {alojam }+\beta_{-} 5 * \text { Busqueda_inst }+\beta_{-} 6 * \text { Superhost }+\beta_{-} 7 * \text { Piscina } \\
& +\beta \_8 * \text { Wif } i+\beta \_9 * \text { Parking_gratis }+\beta \_10 * \text { Ascensor }+\beta \_11 \\
& \text { * Bañera_hidro }+\beta \_12 * \text { Fumar_sí }+\beta \_13 * \text { Mascotas_sí }+\beta \_14 \\
& \text { *Valoración_host }+\beta \_15 * N u m \_r e s e n ̃ a s+u
\end{aligned}
$$


TABLA II

Análisis descriptivo de las variables de estudio

\begin{tabular}{|c|c|c|c|c|c|c|}
\hline VARIABLES & MEDIA & MEDIANA & D.T & MIN. & MAX. & DESCRIPCIÓN \\
\hline Precio & 75,29 & 65,50 & 39,54 & 20 & 420 & $\begin{array}{l}\text { Precio del apartamento por } \\
\text { noche en euros }\end{array}$ \\
\hline \multicolumn{7}{|l|}{ Estructurales } \\
\hline N_baños & 1,215 & 1 & 0,428 & 1 & 4 & $\begin{array}{l}\text { Número de baños en cada } \\
\text { apartamento }\end{array}$ \\
\hline N_dormitorios & 1,631 & 1 & 1,043 & 0 & 7 & Número de habitaciones \\
\hline N_camas & 2,697 & 2 & 1,580 & 1 & 15 & Número de camas \\
\hline \multicolumn{7}{|l|}{ Anfitrión } \\
\hline Num_alojam & 13,507 & 4 & 19,485 & 1 & 71 & $\begin{array}{l}\mathrm{N}^{\mathrm{o}} \text { de apartamentos del } \\
\text { anfitrión en la plataforma }\end{array}$ \\
\hline Busqueda_inst & 0,6415 & 1 & 0,479 & 0 & 1 & $\begin{array}{l}\text { Variable binaria. Valor } 1 \\
\text { reserva inmediata del } \\
\text { alojamiento y } 0 \text { en caso } \\
\text { contrario. }\end{array}$ \\
\hline Superhost & 0,1544 & 0 & 0,361 & 0 & 1 & $\begin{array}{l}\text { Variable binaria. Valor } 1 \\
\text { categoría superhost (mínimo } 10 \\
\text { servicios, } 90 \% \text { de } \\
\text { valoraciones ,5 estrellas el } 80 \% \\
\text { de los casos) }\end{array}$ \\
\hline \multicolumn{7}{|l|}{ Comodidades } \\
\hline Piscina & 0,0573 & 0 & 0,2325 & 0 & 1 & $\begin{array}{l}\text { Variable binaria. Valor 1si hay } \\
\text { piscina y } 0 \text { el caso contrario }\end{array}$ \\
\hline Wifi & 0,8999 & 1 & 0,3001 & 0 & 1 & $\begin{array}{l}\text { Variable binaria. Valor } 1 \text { si hay } \\
\text { conexión Wifi y } 0 \text { el caso } \\
\text { contrario }\end{array}$ \\
\hline Parking_gratis & 0,1306 & 0 & 0,337 & 0 & 1 & $\begin{array}{l}\text { Variable binaria. Valor } 1 \text { si el } \\
\text { apartamento tiene parking } \\
\text { gratuito y } 0 \text { en el caso contrario }\end{array}$ \\
\hline Ascensor & 0,6759 & 1 & 0,468 & 0 & 1 & $\begin{array}{l}\text { Variable binaria. Valor } 1 \text { si hay } \\
\text { ascensor y } 0 \text { en caso contrario }\end{array}$ \\
\hline Bañera_hidro & 0,0096 & 0 & 0,097 & 0 & 1 & $\begin{array}{l}\text { Variable binaria. Valor } 1 \text { si hay } \\
\text { bañera de hidromasaje y } 0 \text { en } \\
\text { caso contrario. }\end{array}$ \\
\hline \multicolumn{7}{|c|}{ Reglas del alojamiento } \\
\hline Fumar_sí & 0,2356 & 0 & 0,424 & 0 & $¡ 1$ & $\begin{array}{l}\text { Variable binaria. Valor } 1 \text { si está } \\
\text { permitido fumar y } 0 \text { en caso } \\
\text { contrario. }\end{array}$ \\
\hline Mascotas_sí & 0,1448 & 0 & 0,352 & 0 & 1 & $\begin{array}{l}\text { Variable binaria. Valor } 1 \text { si } \\
\text { están permitidas mascotas y } 0 \\
\text { en caso contrario. }\end{array}$ \\
\hline \multicolumn{7}{|c|}{ Opiniones/valoraciones } \\
\hline Valoración_host & 0,8936 & 1 & 0,297 & 0 & 1 & $\begin{array}{l}\text { Valoraciones recibidas por los } \\
\text { anfitriones. Escala } 0-1 \text {, dónde } \\
\text { el } 1 \text { es la valoración máxima. }\end{array}$ \\
\hline Num_reseñas & 19,83 & 7 & 33,29 & 0 & 454 & $\begin{array}{l}\text { Numero de comentarios } \\
\text { recibidos por los anfitriones }\end{array}$ \\
\hline
\end{tabular}

Resultados Fuente: Elaboración propia (2018). 


\subsection{Análisis de los resultados}

Para la obtención de un resultado final, durante el proceso se han desarrollado cinco modelos diferentes. En ellos se analiza el efecto de los 5 grupos de variables frente a la endógena de tal manera que: Mod1 (vs. estructurales), Mod2 (Mod1 + anfitrión), Mod3 (Mod2 + comodidades), Mod4 (Mod3 + reglas del alojamiento) y finalmente Mod5 (Mod4 + opiniones/valoraciones) En la Tabla III se presentan los resultados de estos modelos especificados.

En el Mod1 se incluyen las variables explicativas englobadas dentro de la categoría estructural. Todas las variables son significativas y tienen los signos esperados, afectando positivamente sobre la variable endógena. Este modelo tiene un R2 ajustado de 0,2708. Para el siguiente modelo (Mod2) se añaden las variables de la categoría de anfitrión aportando un incremento alrededor de 3\% en el R2 ajustado En esta categoría tenemos variables muy interesantes como es el estatus de superhost, elemento que favorece el incremento del precio. Para el siguiente modelo (Mod3) se han añadido las comodidades y servicios que diferencian los apartamentos de Airbnb. A pesar de que el R2 ajustado aumenta y que el criterio AIC nos indica que seguimos mejorando nuestro modelo, con la inclusión de esta categoría se observa que ciertas variables no son significativas (Piscina, Parking_gratis) afectando incluso al estatus de superhost anterior que tampoco sería significativo en este caso.

Con el Mod4, el cambio apenas se ve reflejado. Con un incremento de medio punto en el R2 ajustado la inclusión de las reglas del alojamiento (ambas variables significativas) no cambian el resultado anterior. Sin embargo, la inclusión de la siguiente categoría sí implica una mejora sustancial en el modelo, aumentando en casi un 4\% el R2 ajustado. Las opiniones y valoraciones han sido defendidas por Airbnb como un aspecto clave en el desarrollo de su plataforma pero el signo de las mismas no es el esperado. Más adelante analizaremos el efecto de esta categoría con más detalle. Respecto al AIC, al igual que ha pasado durante el desarrollo de todo el modelo el resultado ha sido descendente a lo largo del mismo, siendo el valor más pequeño el del Modelo 5 y será el que se analizará con un mayor detalle. Por ello, en el modelo final (Mod5) se ha comprobado el cumplimiento de las hipótesis básicas del modelo de regresión. Así, en relación a la multicolinealidad el número de condición es de 12,4 que al ser menor que 30, podemos afirmar que no hay problemas de multicolinealidad grave. Por otra parte, se ha analizado la hipótesis de normalidad de los residuos. El test de Jarque Bera $(1,04838 \mathrm{y}$ p-valor $=0,5920)$ nos indica que no podemos rechazar la hipótesis nula de normalidad.

Siguiendo el mismo criterio que a lo largo del estudio, analizaremos por categorías los resultados, teniendo en cuenta que al tratarse de un modelo log-lin las variaciones en las variables explicativas supondrán incrementos/decrecimientos porcentuales en la endógena. Con respecto a la categoría estructural, todas las variables son significativas además de ser la categoría más influyente de todas las analizadas, ya que tiene una presencia bastante fuerte en el R2 y es clave desde el inicio para la construcción del modelo. Por ello esta categoría fue elegida como punto de partida. El signo de los resultados es el esperado, con una influencia positiva en el precio ante aumentos en cualquiera de las variables. Sin embargo, el peso no es el mismo, ya que tanto el número de baños como el número de dormitorios tiene más influencia que el número de camas $(14,54 \%$ y $10,97 \%$ respectivamente). Este aspecto es bastante lógico ya que generalmente un mayor número de habitaciones (de cualquier tipo) tienen una influencia positiva bastante alta sobre el precio. (Dubin, 1998).

En lo que se refiere a las características del anfitrión, también todas las variables son significativas al $99 \%$ de confianza pero a diferencia de la categoría anterior el efecto sobre la variable endógena es diferente según la variable. Tanto para el número de alojamientos en la plataforma por anfitrión como para la categoría de superhost el efecto es positivo en el precio. Así, cuando un anfitrión tiene el estatus de superhost, implica un incremento sobre el precio de alquiler del $6,5 \%\left(\left(\mathrm{e}^{\wedge} 0,0636-1\right)^{*} 100\right)$, ceteris paribus. Esto concuerda con investigaciones anteriores en los que este rango está asociado a precios altos (Wang \& Nicolau, 2016). Por otro lado, la variable Búsqueda_inst afecta negativamente al precio, siendo más barato el precio por noche en el caso de que la reserva del alojamiento se pueda realizar sin consentimiento previo del anfitrión. Esto parece razonable, ya que los anfitriones que quieren dar a conocer su alojamiento 
se basan en dos puntos fuertes: los precios más bajos y la facilidad de reservas. (Wang \& Nicolau, 2016).

TABLA III

Resultados de los modelos estimados por MCO. Nota: los p-valores entre paréntesis

\begin{tabular}{|c|c|c|c|c|c|}
\hline & MOD1 & MOD2 & MOD3 & MOD4 & MOD5 \\
\hline Constante & $\begin{array}{c}3,7033 \\
(0,0000)\end{array}$ & $\begin{array}{c}3,7052 \\
(0,0000)\end{array}$ & $\begin{array}{c}3,608 \\
(0,0000)\end{array}$ & $\begin{array}{c}3,6446 \\
(0,0000)\end{array}$ & $\begin{array}{c}3,7755 \\
(0,0000)\end{array}$ \\
\hline \multicolumn{6}{|l|}{ Estructurales } \\
\hline N_baños & $\begin{array}{c}0,1767 \\
(0,0000)\end{array}$ & $\begin{array}{c}0,1561 \\
(0,0000)\end{array}$ & $\begin{array}{c}0,1417 \\
(0,0000)\end{array}$ & $\begin{array}{c}0,1367 \\
(0,0000)\end{array}$ & $\begin{array}{c}0,1358 \\
(0,0000)\end{array}$ \\
\hline N_dormitorios & $\begin{array}{c}0,1097 \\
(0,0000)\end{array}$ & $\begin{array}{c}0,1144 \\
(0,0000)\end{array}$ & $\begin{array}{c}0,1129 \\
(0,0000)\end{array}$ & $\begin{array}{c}0,1136 \\
(0,0000)\end{array}$ & $\begin{array}{c}0,1041 \\
(0,0000)\end{array}$ \\
\hline N_camas & $\begin{array}{c}0,0389 \\
(0,0000)\end{array}$ & $\begin{array}{c}0,0402 \\
(0,0000)\end{array}$ & $\begin{array}{c}0,0428 \\
(0,0000)\end{array}$ & $\begin{array}{c}0,0442 \\
(0,0000)\end{array}$ & $\begin{array}{c}0,0462 \\
(0,0000)\end{array}$ \\
\hline \multicolumn{6}{|l|}{ Anfitrión } \\
\hline Num_alojam & & $\begin{array}{c}0,0042 \\
(0,0000)\end{array}$ & $\begin{array}{c}0,004 \\
(0,0000)\end{array}$ & $\begin{array}{c}0,0037 \\
(0,0000)\end{array}$ & $\begin{array}{c}0,0036 \\
(0,0000)\end{array}$ \\
\hline Búsqueda_inst & & $\begin{array}{l}-0,0784 \\
(0,0000)\end{array}$ & $\begin{array}{l}-0,0876 \\
(0,0000)\end{array}$ & $\begin{array}{l}-0,0902 \\
(0,0000)\end{array}$ & $\begin{array}{l}-0,0685 \\
(0,0000)\end{array}$ \\
\hline Superhost & & $\begin{array}{c}0,0375 \\
(0,0371)\end{array}$ & $\begin{array}{c}0,0245 \\
(0,1723)\end{array}$ & $\begin{array}{c}0,0110 \\
(0,5391)\end{array}$ & $\begin{array}{c}0,0636 \\
(0,0004)\end{array}$ \\
\hline \multicolumn{6}{|l|}{ Comodidades } \\
\hline Piscina & & & $\begin{array}{c}0,0095 \\
(0,7409)\end{array}$ & $\begin{array}{c}0,0035 \\
(0,9019)\end{array}$ & $\begin{array}{c}-0,0130 \\
(0,6407)\end{array}$ \\
\hline Wifi & & & 0,0721 & 0,0665 & 0,1110 \\
\hline \multirow[t]{2}{*}{ Parking_gratis } & & & $-0,0166$ & $-0,0122$ & $-0,0128$ \\
\hline & & & $(0,4037)$ & $(0,5373)$ & $(0,5078)$ \\
\hline \multirow[t]{2}{*}{ Ascensor } & & & 0,0778 & 0,0764 & 0,0708 \\
\hline & & & $(0,0000)$ & $(0,0000)$ & $(0,0000)$ \\
\hline \multirow[t]{2}{*}{ Bañera_hidro } & & & 0,3522 & 0,3421 & 0,3544 \\
\hline & & & $(0,0000)$ & $(0,0000)$ & $(0,0000)$ \\
\hline \multicolumn{6}{|c|}{ Reglas alojamiento } \\
\hline Fumar_sí & & & & $\begin{array}{c}-0,0349 \\
(0,0245)\end{array}$ & $\begin{array}{c}-0,0330 \\
(0,0288)\end{array}$ \\
\hline Mascotas_sí & & & & $-0,0914$ & $-0,0873$ \\
\hline \multicolumn{6}{|c|}{ Opin./valoraciones } \\
\hline \multicolumn{2}{|l|}{ Valoración_host } & & & & $-0,1548$ \\
\hline & & & & & $(0,0000)$ \\
\hline \multirow{2}{*}{ Num_reseñas } & & & & & $-0,0018$ \\
\hline & & & & & $(0,0000)$ \\
\hline $\mathbf{R}^{2}$ & 0,2715 & 0,3053 & 0,3226 & 0,3308 & 0,3667 \\
\hline $\mathbf{R}^{2}$ ajustado & 0,2708 & 0,3039 & 0,3201 & 0,3279 & 0,3634 \\
\hline AIC & 2272,5 & 2137,6 & 2070,9 & 2040,4 & 1881,2 \\
\hline
\end{tabular}

Fuente: Elaboración propia (2018).

En tercer lugar, la categoría de comodidades y servicios es la más amplia, compuesta en su totalidad por variables dicotómicas que tienen un efecto positivo sobre el precio de alquiler. Así, las variables Bañera_hidro tiene un gran efecto positivo sobre los precios $(46,53 \%)$. Por otro lado, otras variables como Ascensor, Parking gratis o Wifi tienen un menor efecto sobre dichos precios. De hecho, a pesar de que la mayoría de los apartamentos tienen conectividad inalámbrica (véase Tabla II), la variable es significativa en el modelo. Sin embargo, en esta categoría la variable Piscina ni tiene el signo esperado ni es significativa.

Con respecto a las reglas del alojamiento, los resultados son confusos. De hecho, la variable Fumar_sí no es significativa. Se está desarrollando un movimiento en Airbnb en torno a la consecución de una tarifa más cara por parte de los anfitriones por permitir fumar en el 
alojamiento (Kennedy, Douglas, Stehouwer, \& Dawson, 2018) pero por otro lado Wang \& Nicolau (2016) catalogan en su estudio a nivel intercontinental que la permisión para fumadores estaba asociada a rangos de precios bajos. La segunda variable (Mascotas_sí) presenta una influencia negativa sobre el precio. Por lo tanto, aquellas viviendas que permiten mascotas son un 9,2\% más baratas. Aquellos propietarios que tienen viviendas con un mayor precio suelen ser más prohibitivos a la hora tanto de aceptar huéspedes como de imponer condiciones en sus alojamientos. Por ello tiene sentido que el segmento de propietarios que acepten mascotas sea aquel que ofrece su alojamiento a un menor precio.

El rasgo más distintivo de Airbnb reside en la categoría de opiniones y valoraciones. Tal y como defiende la propia plataforma, este campo es un aspecto fundamental para el buen funcionamiento. Generalmente, un mayor nivel de ambos está asociado a un precio más elevado. De hecho, para poder llegar a ser superhost es necesario un buen resultado en lo mencionado. Sin embargo, el efecto de ambas variables (Num_reseñas y Valoración_host) no es el esperado ya que presentan una influencia negativa sobre el precio y más con valores tan fuertes como el de Valoración_host (17,11\%). Para el número de comentarios este aspecto se puede deber a que aquellos anfitriones que alquilan a un precio más barato pueden tener una mayor demanda por lo que generan una mayor cantidad de comentarios (Gibbs, Guttentag, Gretzel, Morton, \& Goodwill, 2018), mientras que para las valoraciones puede ser un matiz de percepciones.

En general, como vimos en la tabla 2 las valoraciones suelen ser bastante altas en la plataforma, si a eso le añadimos que puede haber huéspedes que tengan expectativas menores en aquellos alojamientos con precios bajos, puede darse este resultado. (Wang \& Nicolau, 2016) Empíricamente, podemos estudiar la relación entre cada una de estas variables con la endógena mediante la matriz de correlaciones. Como se puede observar en la Tabla 4, existe una correlación negativa tanto de Valoración_Host $(-0,1197)$ como de Num_reseñas $(-0,2247)$ por lo que entendemos que el signo que apreciamos en el modelo es el adecuado.

TABLA IV

Matriz de correlaciones_Log_precio, Valoración_host, Num_reseñas

\begin{tabular}{|l|c|c|c|}
\hline & Log_precio & Valoración_host & Num_reseñas \\
\hline Log_precio & 1,0000 & $-0,1197$ & $-0,2247$ \\
\hline Valoración_host & & 1,0000 & 0,1604 \\
\hline Num_reseñas & & & 1,0000 \\
\hline
\end{tabular}

\subsection{Análisis exploratorio espacial}

Dada la naturaleza de nuestra base de datos y al ver que el aspecto localizativo puede ser importante en este caso, nos ha llevado a analizar la posible presencia de autocorrelación en el precio del alquiler, la cual estaría motivada por la existencia de dependencia espacial en nuestro fenómeno. Esta dependencia espacial se podría deber a la existencia de variables del entorno y de contagio que provocan que alojamientos próximos en el espacio tomen valores similares. Ya que en las investigaciones realizadas en ciencias sociales se suelen formular modelos de carácter general, que luego se pueden o no ajustar con certeza a diferentes casos particulares (Celemín, 2009), es conveniente hacer énfasis en el complemento local para añadir robustez al desarrollo de los modelos más generales. Por ello, se han realizado dos tipos de tests para analizar la presencia de dependencia espacial, uno de tipo global y otro local. En primer lugar, se ha procedido a analizar la presencia de autocorrelación espacial global mediante el estadístico I de Moran:

$$
I=\frac{N}{\sum_{i} \sum_{j} w_{i j}} \frac{\sum_{i} \sum_{j} w_{i j}\left(X_{i}-\bar{X}\right)\left(X_{j}-\bar{X}\right)}{\sum_{i}\left(X_{i}-\bar{X}\right)^{2}}
$$

$\mathrm{N}: \mathrm{n}^{\mathrm{o}}$ de observaciones

$\mathrm{X}$ : variable de interés (Log_precio) 


\section{$\bar{X}$ : media de la variable analizada ( $\log \_$precio)}

$w_{i j}$ : Matriz de pesos espaciales que considera la vecindad de primer orden

El resultado de este estadístico oscila entre -1 y 1 . Valores negativos indican que existe autocorrelación espacial negativa, es decir, que viviendas contiguas presentan valores muy diferentes. El valor 0 indica aleatoriedad en los datos. Mientras que valores positivos indican autocorrelación espacial positiva, es decir, viviendas próximas tienen valores muy similares. En nuestro caso, el resultado del estadístico es 0,120822 con un p-valor de 0,001 por lo que existe autocorrelación espacial positiva significativa. Además de analizar la autocorrelación global, se ha analizado la autocorrelación local, mediante la metodología LISA (Local Indicators of Spatial Autocorrelation) que nos permite detectar las viviendas con precios de alquiler altos (bajos) que están próximas a otras con precios altos (bajos), es decir, viviendas high-high y low-low. También permite detectar datos espaciales outliers (high-low y low-high). En la Figura 1 se muestra el mapa de clusters espaciales. Hay 172 viviendas con precios altos que están junto a otras con precios altos, las cuales se encuentran distribuidas fundamentalmente en la zona sur-oeste de la ciudad, en el centro-sur y en las zonas próximas a la costa y el puerto. Por otra parte, hay 191 viviendas con precios bajos junto a otras con precios bajos localizadas fundamentalmente en el centro-norte de la ciudad y 83 bajos-altos y 98 altos-bajos distribuidas por el centro y norte de la ciudad, respectivamente. Todo esto nos indica que los precios del alquiler de las viviendas no están distribuidos aleatoriamente sobre el plano.

\subsection{Discusión}

Como se comentó al principio del estudio, Airbnb es una de las opciones de alojamiento más demandada en los últimos tiempos. Esta plataforma tiene una gran cuota de mercado que ha venido aumentando exponencialmente en los últimos años, y que llega incluso a superar a gigantes turoperadores como Booking (Hosteltour, 2018). Con su filosofía de trabajo que enfatiza en la confianza entre usuarios y huéspedes, esta empresa no ha dejado indiferente a nadie suponiendo incluso que ciertas empresas como Marriott o Accor copien la estrategia de sharing economy (E1 confidencial, 2018).

El desarrollo de un modelo hedónico de precios permite conocer los factores que determinan dichos precios y el grado de influencia de los factores. En nuestro caso, además, se ha realizado una aproximación al estudio de su distribución espacial. En un momento en el que Airbnb ha afectado negativamente al sector hotelero, dónde existe mucha polémica en torno a la localización de los alojamientos que se utilizan para explotación turística sin la licencia necesaria para ello. Analizar los factores que determinan los precios de alquiler puede ser un punto de partida para llevar a cabo la regulación necesaria. De hecho, en Madrid se prevé que para el mes de Octubre se restringirá el alquiler de viviendas de uso turístico sin licencia, además de limitar la ubicación de las mismas en el centro de la ciudad (La razón, 2018).

Sin embargo, uno de los problemas que más preocupan en torno a esta plataforma, es que se está gestando el crecimiento de una nueva burbuja en los precios del alquiler. La irrupción de Airbnb supone que desde zonas residenciales habituales hasta el centro de la ciudad se convierten en regiones con viviendas turísticas, provocando que la oferta de alquiler de larga estancia disminuya y eso unido al plan de ayuda del alquiler del gobierno para ciertos colectivos hace que finalmente se produzca un incremento de los precios. (Voz Pópuli, 2018). De ahí, que clarificar los factores que determinan el precio sea un aspecto clave para el establecimiento de las políticas necesarias.

\section{CONCLUSIÓN Y LÍNEAS FUTURAS}

Este estudio analiza algunos factores que determinan el precio de las viviendas en alquiler de la plataforma Airbnb, dentro del marco incipiente conocido como sharing economy. La economía de la hospitalidad ha generado un marco de negocio en donde particulares (y profesionales) sacan una rentabilidad de habitaciones o viviendas en desuso en localizaciones donde la industria 
hotelera es incapaz de llegar. Málaga, una ciudad que ha crecido en el ámbito turístico mucho en la última década ve como este fenómeno ha irrumpido en su territorio, aumentando la oferta de alojamiento en cantidades exponenciales.

El precio de los hoteles tradicionalmente está condicionado por características como el número de estrellas, reflejado como un símbolo de calidad. Sin embargo, la determinación de precios en sharing economy es completamente diferente, ya que no es de carácter unilateral sino que el precio dependerá en gran medida del huésped y el anfitrión mediante las opiniones y valoraciones que ambos realizan, tal y como se ha visto reflejado en el modelo. En este trabajo se han considerado cinco tipos de características que permiten explicar el precio de alquiler de las viviendas turísticas en Málaga. Así, se ha cuantificado la influencia de algunas de las principales características estructurales, del anfitrión, comodidades y servicios, reglas del alojamiento y las opiniones y valoraciones que diferencian a cada alojamiento de Airbnb. También, se ha constatado la importancia de que un anfitrión sea considerado como superhost en la plataforma.

Por otro lado, se ha realizado un acercamiento al aspecto espacial. Se ha detectado que la variable de interés presenta autocorrelación espacial, lo cual nos indica que los precios de alquiler presentan dependencia espacial la cual puede venir motivada por un efecto de contagio. Los resultados también muestran la presencia de clusters espaciales, con aglomeraciones de viviendas high-high (próximas a la costa) y low-low (más localizadas en el interior). Todo esto pone de manifiesto la necesidad de seguir investigando en el aspecto localizativo de este fenómeno. También, se han discutido las implicaciones sociales del mismo las cuales han ocupado noticiarios y han propiciado ciertos ajustes legales, siendo el precio y la ubicación de los mismos un aspecto clave dentro de la problemática. Sin embargo, este estudio tiene ciertas limitaciones. Así, en relación a los factores que determinan el precio, tanto la variable Fumar_sí como Piscina resultaron no ser significativas, mientras que en estudios anteriores sí lo fueron. Por otra parte, nos encontramos con aspectos difíciles de explicar en torno al número de comentarios y las valoraciones. Empíricamente en nuestro estudio se ha apreciado una correlación negativa con el precio de los apartamentos, lo cual viene avalado con los resultados de otros autores citados, que obtuvieron resultados similares respecto a estas variables. Se propone una investigación más profunda para ese aspecto, ya que para Airbnb las opiniones y las valoraciones son un aspecto muy importante en el desarrollo como anfitrión en la plataforma. En relación a las líneas futuras de investigación, creemos oportuno seguir trabajando en el análisis espacial añadiendo nuevas variables de tipo localizativo y utilizando métodos más adecuados para modelizar fenómenos espaciales. 
FIGURA I:

Mapa de clusters espaciales LISA

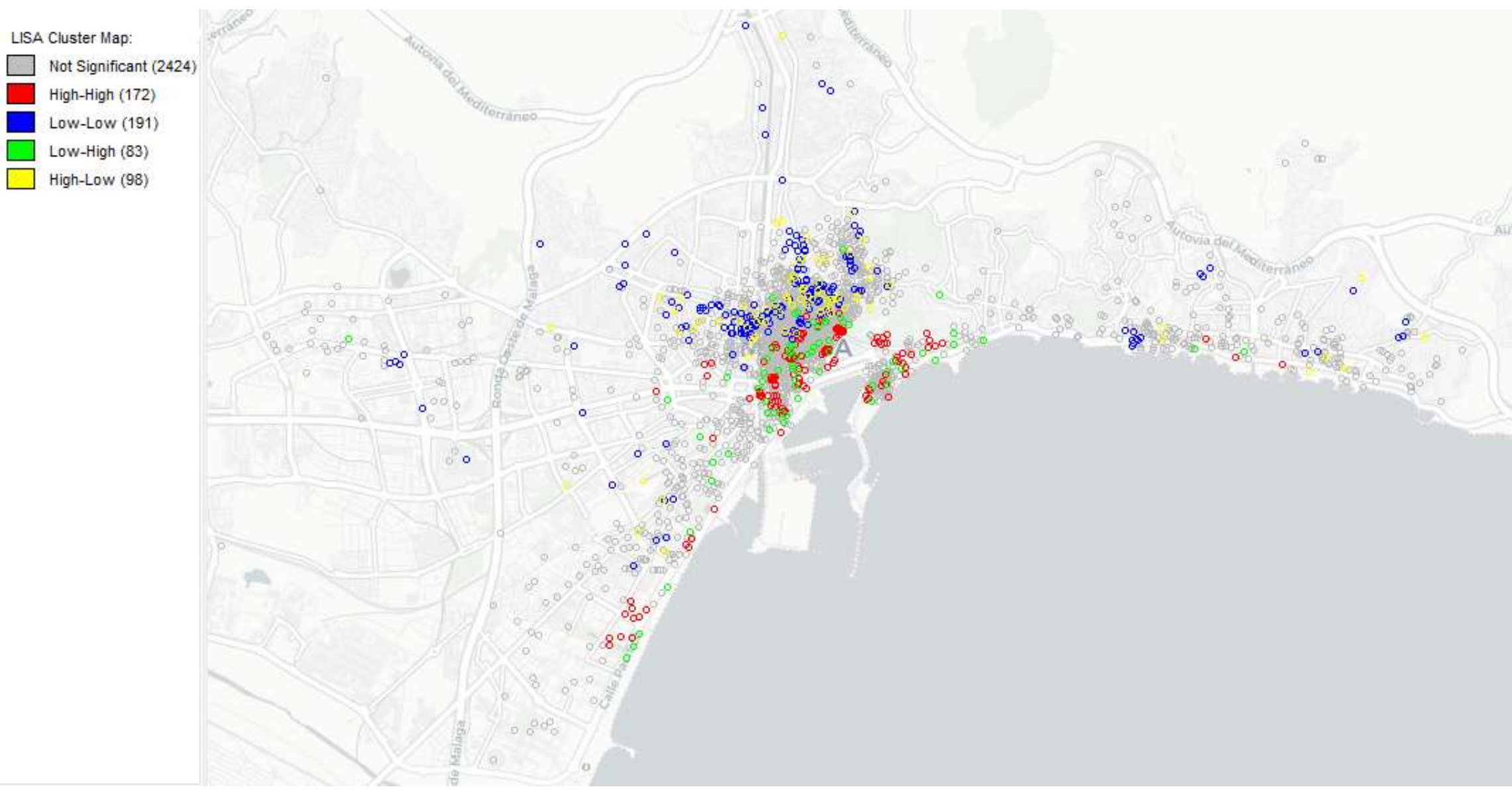




\section{REFERENCIAS BIBLIOGRÁFICAS}

ABDELAZIZ, L., REED, M., TYLER, M., \& YUQING, Z. (2017). Impact of reviews on price: Evidence from sentimental analysis of Airbnb reviews in Boston. Kentucky: Western Kentucky University.

ANSELIN, L. (1995). Local indicators of spatial association - LISA. Geographical analysis, 27(2), 93-115.

BALAGUER, J., \& PERNÍAS, J. (2013). Relationship between spatial agglomeration and hotel prices. Evidence business and tourism consumers. Tourism Management, 36, 391-400.

BELK, R. (2014). You are what you can acces. Sharing and collaborative consumption online. Journal of Business Research, 1595-1600.

BING, F., QIANG, Y., \& ROB, L. (2016). Effect of sharing economy on tourism industry employment. Annals of Tourism Research , 234-378.

CAN, A. (1992). Specification and estimation of hedonic housing price models. Regional Science and Urban Economics, 22, 453-474.

CASE, B., CLAPP, J., DUBIN, R. A., \& RODRIGUEZ, M. (2004). Modeling Espacial and Temporal House Price Patterns: A comparison of Four Models. Journal of Real Estate Finance and Economics, 29(2), 167-191.

CELEMÍN, J. P. (2009). Autocorrelación espacial e indicadores locales de asociación espacial. Importancia, estructura y aplicación. Revista Universitaria de Geografía, 18(1).

CHEN, C.-F., \& ROTHSCHILD, R. (2010). An application of hedonic pricing analysis to the case of hotel rooms in Taipei. Tourism Economics, 16, 685-694.

CHICA OLMO, J., CANO GUERVOS, R., \& CHICA OLMO, M. (2007). Modelo hedónico espacio-temporal y análisis variográfico del precio de la vivienda. Geofocus (Artículos), 7, 56-72.

CUSUMANO, M. A. (2015). How traditional Firms Must Compete in the Sharing Economy. Communications of the ACM, 32-34.

DUBIN, R. A. (1998). Predicting House Prices Using Multiple Listings Data. Journal of Real Estate Finance and Economics, 17(1), 35-59.

El confidencial. (Mayo de 2018). Obtenido de https://www.elconfidencial.com/empresas/2018-05-25/hotelesairbnb-turismo-apartamentos-turisticos_1568670/

GIBBS , C., GUTTENTAG, D., GRETZEL, U., MORTON, J., \& GOODWILL, A. (2018). Pricing in the sharing economy: a hedonic pricing model applied to Airbnb listings. Journal of Travel \& Tourism Marketing, 35, 46-56.

GOBIERNO DE ESPAÑA. MINISTERIO DE ENERGÍA, T. Y. (Mayo de 2018). Encuesta de movimientos turísticos en fronteras (Frontur). Obtenido de http://estadisticas.tourspain.es/eses/estadisticas/frontur/paginas/default.aspx

GUNTER, U. (2018). What makes an Airbnb host a superhost? Empirical evidences for San Francisco and the Bay Area. Tourism Management,66, 26-37.

GUTTENTAG, D. (2015). Airbnb: sisruptive innovation and the rise of an informal tourism accommodation sector. Current Issues in Tourism, 1192-1217.

GUTTENTAG, D. A., \& SMITH, S. J. (2017). Assesing Airbnb as a disruptive innovation relative to hotels: Substitution and comparative performance expectations. International Journal of Hospitality Management, 64, $1-10$.

HAMARI, J., SJÖKLINT, M., \& UKKONEN, A. (2016). The Sharing Economy: Why People Participate in Collaborative Consumption. Journal of the Association for Information Science and Technology, 2047-2059.

HOSTELTOUR. (Mayo de 2018). Obtenido de https://www.hosteltur.com/128256_airbnb-imparable-ya-superabooking-trafico.html

HUNG, W.-T., SHANG, J.-K., \& WANG, F.-C. (2010). Pricing determinants in the hotel industry. Quantile regression analysis. International Journal of Hospitality Management, 29, 378-384.

INSIDE AIRBNB. (2017). Obtenido de Adding data to the debate: http://insideairbnb.com/

JUN, L., MORENO, A., \& ZHANG, D. J. (2015). Agent Behavior in the Sharing Economy: Evidence from Airbnb. Michigan: Michigan Ross.

KENNEDY, R. D., DOUGLAS, O., STEHOUWER, L., \& DAWSON, J. (2018). The availability of smoking-permitted accomodations from Airbnb in 12 Canadian cities. Tobacco Control, 27(1), 112-116.

La razón. (Mayo de 2018). Obtenido de https://www.larazon.es/local/madrid/plan-anti-airbnb--un-90-de-pisosseran-ilegales-desde-octubre-KJ18330129

LEIGHTON, P. (2016). Professional self-employment, new power and the sharing economy: Some cautionary tales from Uber. Journal of Management \& Organization, 859-874.

LIANG, L. J., CHOI, H. C., \& JOPPE, M. (2018). Exploring the relationship between satisfaction, trust and switching intention, repurchase intention in the context of Airbnb. International Journal of Hospitality Management, 41-48.

Libre Mercado. (2015). Obtenido de https://www.libremercado.com/2015-04-09/bombazo-airbnb-ya-es-laempresa-de-alojamiento-mas-grande-del-mundo-1276544991/

MAKARAND , A. M., SUESS, C., \& XINRAN, L. (2017). The accommodation experiencescape: a comparative assessment of hotels and Airbnb. International Journal of Contemporary Hospitality Management, 29, 2377 2404.

Málaga Turismo. (Mayo de 2017). Obtenido de http://www.malagaturismo.com/

MASIERO, L., NICOLAU, J. L., \& LAW, R. (2015). A demand-driven analysis of tourist accomodation price: A quantile regression of room bookings. International Journal of Hospitality Management, 50, 1-8. 
MILLER, S. R. (2016). FIRST PRINCIPLES FOR REGULATING THE SHARING ECONOMY. Harvard Journal of Legislation, 147-202.

MÖHLMANN, M. (2015). Collaborative consumption: determinants of satisfaction and the likelihood of using a sharing economy option again. Journal of Consumer Behaviour, 193-207.

MORGAN, B., \& KUCH, D. (2015). Radical Transactionalism: Legal Consciousness, Diverse Economies, and the Sharing Economy. Journal of Law and Society, 556-587.

MURILLO FORT, C., \& GONZÁLEZ LOPEZ-VALCÁRCEL, B. (2000). Manual de econometría.

ROSEN, S. (1974). Hedonic Price and Implicit Markets: Product Differentiation in Pure Competition. Journal of Political Economy, 82, 34-55.

SCHWALBE, U. (2018). Creative destruction and the sharing economy: Uber as disruptive innovation. Journal of Economics, 93-96.

SHAHEEN, S., STOCKER, A., \& MUNDLER, M. (2017). Online and App-Based Carpooling in France: Analyzing Users and Practices - A Study of Blablacar. En G. Meyer, \& S. Shaheen, Disrupting Mobility. Lecture Notes in Mobility (págs. 181-196). Springer International Publishing .

SO, K. F., OH, H., \& MIN, S. (2018). Motivations and constraints of Airbnb consumers: Findings from a mixedmethods approach. Tourism Management, 224-236.

THORNE, D. M., \& QUINN, F. (2017). Supplier Resources in the Sharing Economy: Three Regulatory Concerns. Journal of Marketing Channels, 73-83.

THRANE, C. (2007). Examining the determinants of room rates for hotels in capital cities: The oslo experience. Journal of Revenue \& Pricing Management,5, 315-323.

Voz Pópuli. (Mayo de 2018). Obtenido de https://www.vozpopuli.com/economia-y-finanzas/empresas/Haciaburbuja-politicos-fondos-Airbnb-disparan-precio-alquiler_0_1123387972.html

WANG, D., \& NICOLAU, J. L. (2016). Price determinants of sharing eocnomy based accommodation rental: A study of listing from 33 cities on Airbnb.com. International Journal of Hospitality Management, 120-131.

WHITE, H. (1980). A heteroskedasticity-consistent covariance matrix estimator and a direct test for heteroskedasticity. Journal of econometric society, 48(4), 817-838.

World Tourism Organization (UNWTO). (Mayo de 2018). Obtenido de http://publications.unwto.org/

YOONJOUNG HEO, C. (2016). Sharing economy and prospects in tourism research. Annals of Tourism Research, 156-170.

ZERVAS, G., PROSERPIO, D., \& BYERS, J. W. (2017). The Rise of the Sharing Economy: Estimating the Impact of Airbnb on the Hotel Industry. Journal of Marketing Research, 54, 687-705.

ZHANG, H., ZHANG, J., LU, S., CHENG, S., \& ZHANG, J. (2011). Modeling hotel room price with geographically weighted regression. International Journal of Hospitality Management, 30, 1036-1043.

ZHANG, Z., CHEN, R. C., HAN, L. D., \& YANG, L. (2017). Key factors affecting the price of Airbnb listings: a geographically weighted approach. Sustainablity, 9, 1635. 
